\title{
Determination of the Frumkin and Temkin Adsorption Isotherms of Under- potentially Deposited Hydrogen at Pt Group Metal Interfaces Using the Standard Gibbs Energy of Adsorption and Correlation Constants
}

\author{
Jinyoung Chun ${ }^{1}$, Sang K. Jeon ${ }^{2}$, and Jang H. Chun*,3 \\ ${ }^{1}$ Department of Chemical Engineering, Pohang University of Science and Technology, Pohang, Kyungbuk 790-784, \\ Republic of Korea \\ ${ }^{2}$ International Cooperation Division, Korea Institute ofLighting Technology, Bucheon, Kyunggi 420-806, Republic ofKorea \\ ${ }^{3}$ Department of Electronic Engineering, Kwangwoon University, Seoul 139-701, Republic of Korea
}

(Received November 29, 2013 : Accepted November 30, 2013)

\begin{abstract}
At Pt(111), Pt(100), Pt, and Rh interfaces, the Frumkin adsorption isotherm of underpotentially deposited hydrogen (UPD H) and related electrode kinetic data are determined using the standard Gibbs energy of adsorption. The Temkin adsorption isotherm of UPD H correlating with the Frumkin adsorption isotherm of UPD $\mathrm{H}$ is readily determined using the correlation constants between the Temkin and Frumkin or Langmuir adsorption isotherms. At the $\operatorname{Pt}(111)$, $\mathrm{Pt}(100)$, Pt, and $\mathrm{Rh}$ interfaces, the lateral repulsive interaction between the UPD $\mathrm{H}$ species is interpreted using the interaction parameter for the Frumkin adsorption isotherm. The lateral repulsive interaction between the UPD $\mathrm{H}$ species at the $\mathrm{Pt}(111), \mathrm{Pt}(100), \mathrm{Pt}$, and $\mathrm{Rh}$ interfaces is significantly different from the lateral attractive interaction between the overpotentially deposited hydrogen $(\mathrm{OPD} H)$ species at Pt, Ir, and Pt-Ir alloy interfaces.
\end{abstract}

Key words : Underpotentially and overpotentially deposited hydrogen; Frumkin, Langmuir, and Temkin adsorption isotherms; Pt group metal electrodes; Standard Gibbs energy of adsorption; Correlation constants

\section{Introduction}

It is well known that underpotentially deposited hydrogen (UPD H) and overpotentially deposited hydrogen (OPD H) occupy different surface adsorption sites and act as two distinguishable electroadsorbed $\mathrm{H}$ species, and that only OPD $\mathrm{H}$ can contribute to the cathodic $\mathrm{H}_{2}$ evolution reaction (HER). ${ }^{1-5)}$ Although the Frumkin and Langmuir adsorption isotherms may be regarded as classical models and theories, it is preferable to consider the Frumkin and Langmuir adsorption isotherms for UPD $\mathrm{H}$ and OPD $\mathrm{H}$ rather than equations of the electrode kinetics and thermodynamics for UPD $\mathrm{H}$ and OPD $\mathrm{H}$ because these adsorption isotherms are associated more directly with the atomic mecha-

*E-mail: jhchun@kw.ac.kr nisms of UPD $\mathrm{H}$ and OPD $\mathrm{H}^{6}{ }^{6}$ However, there is not much reliable information on the Frumkin and Temkin adsorption isotherms of UPD $\mathrm{H}$ and OPD $\mathrm{H}$ and related electrode kinetic data, i.e., the fractional surface coverage vs. potential ( $\theta$ vs. $E$ ), interaction parameter $(\mathrm{g})$, and equilibrium constant $(K)$. A quantitative relationship between the Temkin and Frumkin or Langmuir adsorption isotherms of UPD $\mathrm{H}$ and OPD $\mathrm{H}$ has not been developed to study the cathodic HER. Note that the Frumkin adsorption isotherms of UPD $\mathrm{H}$ and OPD $\mathrm{H}$ and related electrode kinetic data ( $\theta$ vs. $E$, g, $K$ ) have never been experimentally determined using conventional methods. ${ }^{2,3)}$ Therefore, the thermodynamic data for UPD H, i.e., the standard Gibbs energy of adsorption $\left(\Delta G_{\theta}{ }^{\circ}\right)$ and rate of change $(r)$ of $\Delta G_{\theta}{ }^{\circ}$ with $\theta(0 \leq \theta \leq 1)$, have been frequently investigated and reported. ${ }^{1-5)}$ 
To determine the Frumkin, Langmuir, and Temkin adsorption isotherms of UPD $\mathrm{H}$ and OPD $\mathrm{H}$, the phase-shift method and correlation constants have been originally developed on the basis of relevant experimental results and data. ${ }^{7)}$ The theoretical and experimental backgrounds and basic procedure of the phase-shift method for determining the Frumkin, Langmuir, and Temkin adsorption isotherms are described elsewhere. ${ }^{8)}$

In this paper, we determined the electrode kinetic data $(\mathrm{g}, K)$ for the Frumkin adsorption isotherms $(\theta$ vs. $E$ ) of UPD $\mathrm{H}$ at $\mathrm{Pt}(111), \mathrm{Pt}(100), \mathrm{Pt}$, and $\mathrm{Rh} /$ aqueous solution interfaces using $\Delta G_{\theta}{ }^{0}$ with $\theta^{2-5)}$ The Temkin adsorption isotherms of UPD $\mathrm{H}$ correlating with the Frumkin adsorption isotherms of UPD $\mathrm{H}$ were readily determined using the correlation constants. ${ }^{7,8)}$ The Frumkin and Temkin adsorption isotherms of UPD $\mathrm{H}$ and electrode kinetic data ( $\theta$ vs. $E, \mathrm{~g}, K$ ) have never been determined using $\Delta G_{\theta}{ }^{\circ}$ with $\theta(0 \leq \theta \leq 1)$ and the correlation constants.

\section{Determination of the Frumkin adsorption isotherm using the standard Gibbs energy of adsorption}

Under the Frumkin adsorption conditions, the relationship between the equilibrium constant $(K)$ and the standard Gibbs energy of adsorption $\left(\Delta G_{\theta}{ }^{\circ}\right)$ is ${ }^{9)}$

$$
\begin{aligned}
& 2.3 R T \log K=-\Delta G_{\theta}{ }^{\circ} \\
& K=K_{\mathrm{o}} \exp (-\mathrm{g} \theta) \\
& \mathrm{g}=r / R T
\end{aligned}
$$

where $R$ is the gas constant, $T$ is the absolute temperature, $K$ is the equilibrium constant for the Frumkin adsorption isotherm, $\Delta G_{\theta}{ }^{\circ}$ is the standard Gibbs energy of adsorption with $\theta(0 \leq \theta \leq 1), K_{\mathrm{o}}$ is the equilibrium constant for the Frumkin adsorption isotherm at $\mathrm{g}=0$, $\mathrm{g}$ is the interaction parameter for the Frumkin adsorption isotherm, $\theta(0 \leq \theta \leq 1)$ is the fractional surface coverage, and $r$ is the rate of change of $\Delta G_{\theta}{ }^{\circ}$ with $\theta(0 \leq \theta \leq 1)$. The dimension of $K$ is described elsewhere. ${ }^{10)}$ The electrode kinetic and thermodynamic data (g, $r$,
Table 1. Comparison of the interaction parameters (g), rates of change $(r)$ of $\Delta G_{\theta}{ }^{0}$ with $\theta$, and standard Gibbs energies of UPD $H\left(\Delta G_{\theta}{ }^{0}\right)$ for the Frumkin adsorption isotherms at the Pt group metal interfaces

\begin{tabular}{ccccc}
\hline Interface & $\mathrm{g}$ & $r / \mathrm{kJ} \mathrm{mol}^{-1}$ & $\Delta G_{\theta}{ }^{\circ} / \mathrm{kJ} \mathrm{mol}^{-1}$ & Ref. \\
\hline $\mathrm{Pt}(111) / 0.05 \mathrm{M}$ & 11.0 & 27.3 & $-26 \leq \Delta G_{\theta}{ }^{\circ} \leq-8^{b}$ & 2,3 \\
$\mathrm{H}_{2} \mathrm{SO}_{4}$ & & & $-25 \leq \Delta G_{\theta}{ }^{\circ} \leq-12^{c}$ & 2,3 \\
$\mathrm{Pt} / 0.5 \mathrm{M} \mathrm{H}_{2} \mathrm{SO}_{4}$ & - & - & $-21 \leq \Delta G_{\theta}{ }^{\circ} \leq-7^{c}$ & 2,3 \\
$\mathrm{Pt} / 0.5 \mathrm{M} \mathrm{HClO}_{4}$ & - & - & -21 \\
$\mathrm{Rh} / 0.5 \mathrm{M} \mathrm{H}_{2} \mathrm{SO}_{4}$ & - & - & $-18 \leq \Delta G_{\theta}{ }^{\circ} \leq-8^{c}$ & 2 \\
$\mathrm{Pt}(111) / 0.1 \mathrm{M}$ & 11.2 & 27.5 & $-27 \leq \Delta G_{\theta}{ }^{\circ} \leq-8^{d}$ & 4 \\
$\mathrm{HClO}_{4}$ & & & & \\
$\mathrm{Pt}(100) / 0.1 \mathrm{M}$ & 4.0 & $9.9^{a}$ & $-38 \leq \Delta G_{\theta}{ }^{\circ} \leq-29^{d}$ & 4 \\
$\mathrm{HClO}_{4}$ & & & - & 5 \\
$\mathrm{Pt} / 0.5 \mathrm{M} \mathrm{H}_{2} \mathrm{SO}_{4}$ & 1.9 & - & - & \\
\hline $\mathrm{Ac}$ & & & \\
\hline
\end{tabular}

$\overline{{ }^{a} \text { Adapted for this work. }{ }^{b} \Delta G_{\theta}{ }^{0} \text { with } \theta(0 \leq \theta \leq 2 / 3) \cdot{ }^{c} \Delta G_{\theta}{ }^{0}}$ with $\theta(0 \leq \theta \leq 1) .{ }^{d} \Delta G_{\theta}{ }^{\circ}$ with $\theta(0.05 \leq \theta \leq 0.6)$. Note that the Frumkin adsorption isotherms are valid and effective at $0 \leq \theta \leq 1$.

$\Delta G_{\theta}{ }^{\circ}$ ) for the Frumkin adsorption isotherms of UPD $\mathrm{H}$ at the $\mathrm{Pt}(111), \operatorname{Pt}(100)$, Pt, and $\mathrm{Rh}$ interfaces are summarized in Table $1 .^{2-5)}$

The Frumkin adsorption isotherm assumes that the lateral interaction effect is not negligible. It is well known that the Langmuir adsorption isotherm is a special case of the Frumkin adsorption isotherm. Note that when $\mathrm{g}=0$ in Eqs. 2 and 3, the Langmuir adsorption isotherm is obtained. For the Langmuir adsorption isotherm, the lateral interaction effect is assumed to be negligible. The Frumkin adsorption isotherm $(\theta$ vs. $E)$ can be expressed as follows ${ }^{9)}$

$$
\left[\frac{\theta}{1-\theta}\right] \exp (g \theta)=K_{\mathrm{o}} C^{+} \exp (-E F / R T)
$$

where $C^{+}$is the concentration of ions (i.e., $\mathrm{H}^{+}$) in the bulk solution, $E$ is the negative potential, and $F$ is Faraday's constant.

The electrode kinetic data $\left(\mathrm{g}, K_{\mathrm{o}}, K\right)$ for the Frumkin adsorption isotherms of UPD $\mathrm{H}$ shown in Table 2 are calculated using Eqs. 1 to 4 and the relevant experimental data shown in Table 1. In Table 2, we assume that the adsorptions of UPD $H$ shown in Table 1 depend on $\theta(0 \leq \theta \leq 1)$ and $E$ regardless of the temperature ranges. The ranges of $\theta$ given in Table 1 (see Footnotes $b$ to $d$ ) do not influence the 
Table 2. Determination of the interaction parameters (g) and equilibrium constants $(K)$ of UPD $H$ for the Frumkin and Temkin adsorption isotherms at the Pt group metal interfaces using the standard Gibbs energies of UPD $H\left(\Delta G_{\theta}{ }^{\circ}\right)$ and the correlation constants

\begin{tabular}{|c|c|c|c|c|c|}
\hline \multirow{2}{*}{ Interface } & \multicolumn{2}{|r|}{ Frumkin } & \multicolumn{2}{|r|}{ Temkin } & \multirow{2}{*}{ Ref. } \\
\hline & $\mathrm{g}$ & $K / \mathrm{mol}^{-1}$ & $\mathrm{~g}$ & $K / \mathrm{mol}^{-1}$ & \\
\hline $\mathrm{Pt}(111) / 0.05 \mathrm{M} \mathrm{H}_{2} \mathrm{SO}_{4}$ & 11.0 & $3.6 \times 10^{4} \exp (-11 \theta)^{b}$ & 15.6 & $3.6 \times 10^{5} \exp (-15.6 \theta)$ & this work \\
\hline $\mathrm{Pt} / 0.5 \mathrm{M} \mathrm{H}_{2} \mathrm{SO}_{4}$ & $5.2^{a}$ & $2.4 \times 10^{4} \exp (-5.2 \theta)^{b}$ & 9.8 & $2.4 \times 10^{5} \exp (-9.8 \theta)$ & this work \\
\hline $\mathrm{Pt} / 0.5 \mathrm{M} \mathrm{HClO}_{4}$ & $5.6^{a}$ & $4.8 \times 10^{3} \exp (-5.6 \theta)^{b}$ & 10.2 & $4.8 \times 10^{4} \exp (-10.2 \theta)$ & this work \\
\hline $\mathrm{Rh} / 0.5 \mathrm{M} \mathrm{H}_{2} \mathrm{SO}_{4}$ & $4.0^{a}$ & $1.4 \times 10^{3} \exp (-4 \theta)^{b}$ & 8.6 & $1.4 \times 10^{4} \exp (-8.6 \theta)$ & this work \\
\hline $\mathrm{Pt}(111) / 0.1 \mathrm{M} \mathrm{HClO}_{4}$ & 11.2 & $5.4 \times 10^{4} \exp (-11.2 \theta)^{b}$ & 15.8 & $5.4 \times 10^{5} \exp (-15.8 \theta)$ & this work \\
\hline $\mathrm{Pt}(100) / 0.1 \mathrm{M} \mathrm{HClO}_{4}$ & 4.0 & $4.6 \times 10^{6} \exp (-4 \theta)^{b}$ & 8.6 & $4.6 \times 10^{7} \exp (-8.6 \theta)$ & this work \\
\hline $\mathrm{Pt} / 0.5 \mathrm{M} \mathrm{H}_{2} \mathrm{SO}_{4}$ & 1.9 & $A \exp (-1.9 \theta)^{c}$ & 6.5 & $10 \mathrm{~A} \exp (-6.5 \theta)$ & this work \\
\hline
\end{tabular}

${ }^{a}$ Calculated using Eq. 3 and the relevant experimental data shown in Table $1 .{ }^{b}$ Calculated using Eqs. 1 to 4 and the relevant experimental data shown in Table $1 .{ }^{c} \mathrm{~A}$ is the equilibrium constant at $\mathrm{g}=0$, i.e. $K_{\mathrm{o}}$. Note that the values of $\mathrm{g}$ and $K_{\mathrm{o}}$ for the Temkin adsorption isotherms are approximately 4.6 and 10 times greater than those for the correlated Frumkin adsorption isotherms, respectively. The Temkin adsorption isotherms are valid and effective only at $0.2<\theta<0.8$.

determination of the Frumkin adsorption isotherms of UPD H. Because only one Frumkin adsorption isotherm of UPD $\mathrm{H}$ is determined regardless of the ranges of $\theta$ over the same potential range. ${ }^{7}$

\section{Determination of the Temkin adsorption isotherm using the correlation constants}

At intermediate values of $\theta$, i.e., $0.2<\theta<0.8$, the pre-exponential term, $[\theta /(1-\theta)]$, in Eq. 4 varies little with $\theta$ in comparison with the variation of the exponential term, $\exp (\mathrm{g} \theta)$. Under these approximate conditions, the Temkin adsorption isotherm can be simply derived from the Frumkin adsorption isotherm. The Temkin adsorption isotherm ( $\theta$ vs.E) can be expressed as follows ${ }^{9)}$

$$
\exp (\mathrm{g} \theta)=K_{\mathrm{o}} C^{+} \exp (-E F / R T)
$$

The physical meaning of $\mathrm{g}$ for the Temkin adsorption isotherm (Eq. 5) is clearly different from that for the Frumkin adsorption isotherm (Eq. 4). Note that $\mathrm{g}$ in Eq. 5 is practically determined by the slope of experimental data for $0.2<\theta<0.8$ (see Figs. 1 to 3 ). The evaluation of the applicability of the Frumkin and Temkin adsorption isotherms over the same potential range is described elsewhere. ${ }^{7)}$

The cathodic HER at the Pt group metal interfaces is one of the most extensively studied topics in elec-

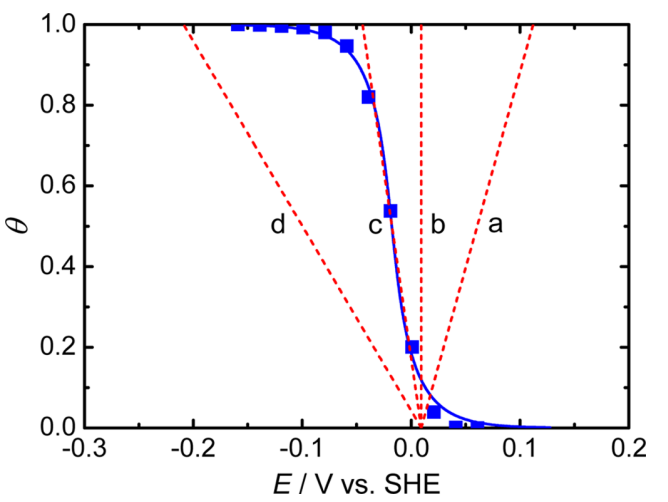

Fig. 1. Comparison of the Frumkin and four fitted Temkin adsorption isotherms at the $\mathrm{Pt} / 0.5 \mathrm{M} \mathrm{H}_{2} \mathrm{SO}_{4}$ aqueous solution interface (adapted from Ref. 11). All potentials $(E)$ are given on the standard hydrogen electrode (SHE) scale. Experimental data: $\square$. The solid curve shows the Frumkin adsorption isotherm, i.e., $K=3.5 \times 10^{-5} \exp (2.5 \theta) \mathrm{mol}^{-1}$, calculated using Eq. 4. Dashed lines show the Temkin adsorption isotherms calculated using Eq. 5 and the correlation constants for (a) $\mathrm{g}=-4$, (b) $\mathrm{g}=0$, (c) $\mathrm{g}=2.1$, and (d) $\mathrm{g}=8.5$ with $K_{0}=3.5 \times 10^{-4} \mathrm{~mol}^{-1}$. Note that the Temkin adsorption isotherm, i.e., $K=3.5 \times 10^{-4} \exp (-2.1 \theta) \mathrm{mol}^{-1}$, is valid and effective only at $0.2<\theta<0.8$.

trochemistry, electrode kinetics, hydrogen energy, and fuel cells, etc. ${ }^{2-5)}$ Figs 1,2 , and 3 show the determination of the Temkin adsorption isotherms of OPD $\mathrm{H}$ corresponding to the Frumkin adsorption isotherms of OPD $\mathrm{H}$ at $\mathrm{Pt}^{11)}{ }^{11}{ }^{11)}$ and $\mathrm{Pd}^{12)}$ interfaces, respectively. The dashed lines in Figs. 1 to 3 show the numerically calculated Temkin adsorption isotherms 


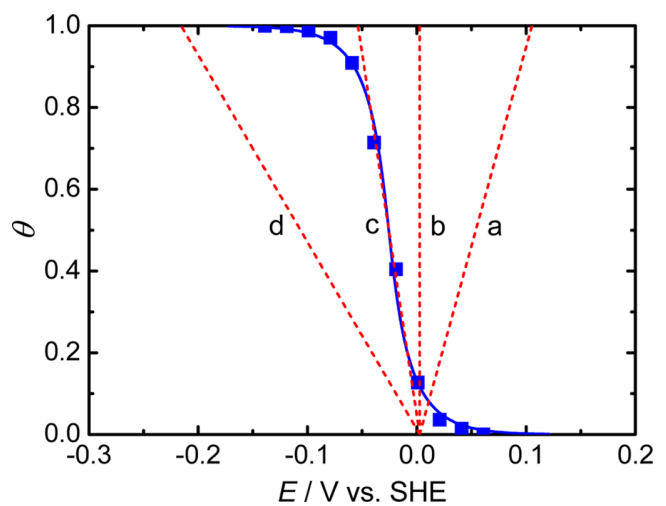

Fig. 2. Comparison of the Frumkin and four fitted Temkin adsorption isotherms at the $\mathrm{Ir} / 0.5 \mathrm{M} \mathrm{H}_{2} \mathrm{SO}_{4}$ aqueous solution interface (adapted from Ref. 11). All potentials $(E)$ are given on the standard hydrogen electrode (SHE) scale. Experimental data: $\square$. The solid curve shows the Frumkin adsorption isotherm, i.e., $K=2.7 \times 10^{-5} \exp (2.4 \theta) \mathrm{mol}^{-1}$, calculated using Eq. 4 . Dashed lines show the Temkin adsorption isotherms calculated using Eq. 5 and the correlation constants for (a) $\mathrm{g}=-4$, (b) $\mathrm{g}=0$, (c) $\mathrm{g}=2.2$, and (d) $\mathrm{g}=8.5$ with $K_{\mathrm{o}}=2.7 \times 10^{-4} \mathrm{~mol}^{-1}$. Note that the Temkin adsorption isotherm, i.e., $K=2.7 \times 10^{-4} \exp (-2.2 \theta) \mathrm{mol}^{-1}$, is valid and effective only at $0.2<\theta<0.8$.

using Eq. 5. The Temkin adsorption isotherms corresponding to the Frumkin adsorption isotherms are labeled c. Note that the values of $\mathrm{g}$ and $K_{\mathrm{o}}$ for the Temkin adsorption isotherms are approximately 4.6 and 10 times greater than those for the correlated Frumkin adsorption isotherms, respectively. The Temkin adsorption isotherms are valid and effective only at $0.2<\theta<0.8$.

As shown in Figs. 1 to 3 , g for the Temkin adsorption isotherm can be simply determined by the slope of experimental data for $0.2<\theta<0.8$. This implies that the two different adsorption isotherms, i.e., the Temkin and Frumkin or Langmuir adsorption isotherms, appear to fit the same data regardless of their adsorption conditions. Correspondingly, the physical meaning of $\mathrm{g}$ for the Temkin adsorption isotherm is clearly different from that for the Frumkin adsorption isotherm regardless of their mathematical approximations. In other words, $\mathrm{g}$ for the Temkin adsorption isotherm (Eq. 5) does not imply the correct lateral interaction between the UPD $\mathrm{H}$ or OPD $\mathrm{H}$ species. This is the reason why the applicability and its limitations of

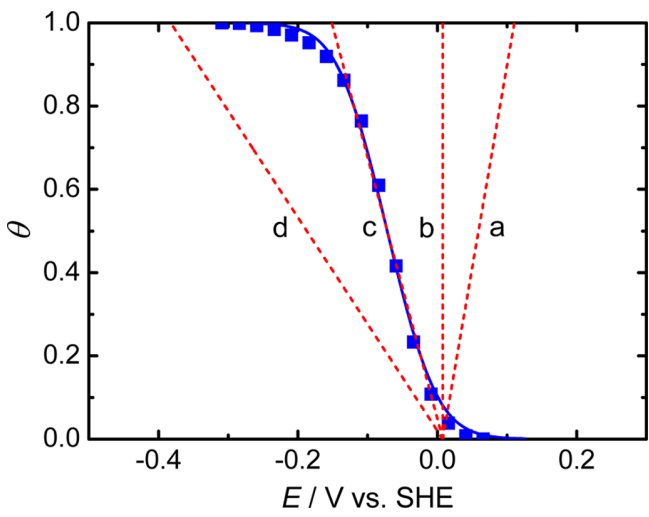

Fig. 3. Comparison of the Frumkin and four fitted Temkin adsorption isotherms at the $\mathrm{Pd} / 0.5 \mathrm{M} \mathrm{H}_{2} \mathrm{SO}_{4}$ aqueous solution interface (adapted from Ref. 12). All potentials $(E)$ are given on the standard hydrogen electrode (SHE) scale. Experimental data: $\square$. The solid curve shows the Frumkin adsorption isotherm, i.e., $K=3.3 \times 10^{-5} \exp (-1.6 \theta) \mathrm{mol}^{-1}$, calculated using Eq. 4 . Dashed lines show the Temkin adsorption isotherms calculated using Eq. 5 and the correlation constants for (a) $g=-4$, (b) $g=0$, (c) $g=6.2$, and (d) $g=15.2$ with $K_{0}=3.3 \times 10^{-4} \mathrm{~mol}^{-1}$. Note that the Temkin adsorption isotherm, i.e., $K=3.3 \times 10^{-4} \exp (-6.2 \theta) \mathrm{mol}^{-1}$, is valid and effective only at $0.2<\theta<0.8$.

the Temkin adsorption isotherms for UPD $\mathrm{H}$ and OPD $\mathrm{H}$ should be considered at the Pt group metal interfaces. The $\mathrm{g}$ and $K$ for the Temkin adsorption isotherms of OPD $\mathrm{H}$ at noble metal interfaces are summarized elsewhere. ${ }^{7)}$

As stated above, we have experimentally and consistently found and confirmed that the equilibrium constants $\left(K_{\mathrm{o}}\right)$ for the Temkin adsorption isotherms are approximately 10 times greater than those $\left(K_{0}\right.$ or $\left.K\right)$ for the correlated Frumkin or Langmuir adsorption isotherms. ${ }^{7,8,11,12)}$ These factors (ca. 4.6 and 10) can be taken as correlation constants between the Temkin and Frumkin or Langmuir adsorption isotherms. Finally, one can conclude that the Temkin adsorption isotherm of UPD $\mathrm{H}$ correlating with the Frumkin or Langmuir adsorption isotherm of UPD $\mathrm{H}$, and vice versa, is readily determined using the correlation constants. The electrode kinetic data $\left(\mathrm{g}, K_{\mathrm{o}}, K\right)$ for the Temkin adsorption isotherms ( $\theta$ vs. $E$ ) of UPD $\mathrm{H}$ at the $\mathrm{Pt}(111), \operatorname{Pt}(100), \mathrm{Pt}$, and $\mathrm{Rh}$ interfaces are readily determined using the correlation constants and are summarized in Table 2. 


\section{Interaction parameter for the Frumkin adsorption isotherm}

At the $\mathrm{Pt}(111), \mathrm{Pt}(100), \mathrm{Pt}$, and $\mathrm{Rh}$ interfaces, the positive values of $\mathrm{g}$ for the Frumkin adsorption isotherms shown in Table 2 imply the lateral repulsive interaction between the UPD $\mathrm{H}$ species, which leads to a decrease in the absolute value of the standard Gibbs energy $\left(\left|\Delta G_{\theta}^{o}\right|\right)$ of UPD H with $\theta$ $(0 \leq \theta \leq 1)$ (see Table 1). Note that $\Delta G_{\theta}{ }^{\circ}$ is a negative number, i.e. $\Delta G_{\theta}{ }^{\mathrm{o}}<0$.

At Pt, Ir, and Pt-Ir alloy interfaces, ${ }^{7)}$ the negative values of $\mathrm{g}$ for the Frumkin adsorption isotherms imply the lateral attractive interaction between the OPD $\mathrm{H}$ species, which leads to an increase in $\left|\Delta G_{\theta}^{o}\right|$ of OPD $\mathrm{H}$ with $\theta(0 \leq \theta \leq 1) .^{9,14)}$ This is a unique feature of OPD $\mathrm{H}$ species at the $\mathrm{Pt}$ group metal interfaces. ${ }^{13)}$ However, the lateral attractive interaction $(\mathrm{g}<0)$ between the OPD $\mathrm{H}$ species at the Pt, Ir, and Pt-Ir alloy interfaces is significantly different from the lateral repulsive interaction $(g>0)$ between the OPD $\mathrm{H}$ species at a Pd interface even though the $\mathrm{Pd}$ belongs to the $\mathrm{Pt}$ group metals..$^{711-13)}$ This aspect is frequently overlooked and confused in the Temkin adsorption isotherm of OPD $\mathrm{H}$ at the Pt group metal interfaces.

\section{Conclusions}

At the $\operatorname{Pt}(111), \operatorname{Pt}(100), \mathrm{Pt}$, and $\mathrm{Rh}$ interfaces, the electrode kinetic data $\left(\mathrm{g}, K_{\mathrm{o}}, K\right)$ for the Frumkin and Temkin adsorption isotherms ( $\theta$ vs. $E$ ) of UPD $\mathrm{H}$ are readily determined using the standard Gibbs energy of adsorption $\left(\Delta G_{\theta}{ }^{\circ}\right)$ and the correlation constants. The lateral repulsive interaction $(\mathrm{g}>0)$ between the UPD $\mathrm{H}$ species at the $\operatorname{Pt}(111), \operatorname{Pt}(100)$, $\mathrm{Pt}$, and $\mathrm{Rh}$ interfaces is significantly different from the lateral attractive interaction $(g<0)$ between the OPD $\mathrm{H}$ species at the Pt, Ir, and Pt-Ir alloy interfaces. The lateral attractive interaction $(\mathrm{g}<0)$ between the OPD $\mathrm{H}$ species is a unique feature of the Pt, Ir, and Pt-Ir alloy interfaces. The lateral repulsive interaction $(g>0)$ between the OPD $\mathrm{H}$ species is a unique feature of the Pd interface.

For $0.2<\theta<0.8$, the two different adsorption isotherms, i.e., the Temkin and Frumkin or Langmuir adsorption isotherms, appear to fit the same data regardless of their adsorption conditions. The physical meaning of $\mathrm{g}$ for the Temkin adsorption isotherm is clearly different from that for the Frumkin adsorption isotherm. The applicability and its limitations of the Temkin adsorption isotherms for UPD $\mathrm{H}$ and OPD $\mathrm{H}$ should be considered at the $\mathrm{Pt}$ group metal/aqueous solution interfaces. The Temkin adsorption isotherm correlating with the Frumkin or Langmuir adsorption isotherm, and vice versa, is readily determined using the correlation constants.

\section{Acknowledgments}

Figures 1 to 3 were reprinted and adapted with permission from the International Journal of Hydrogen Energy 32, 1982 (2007) and 33, 4962 (2008). The authors wish to thank the International Association for Hydrogen Energy (IAHE). This work was supported by the Research Grant of Kwangwoon University in 2013.

\section{References}

1. E. Gileadi, "Electrode kinetics", VCH: New York, 1993.

2. B. E. Conway and G. Jerkiewicz, Eds., "Electrochemistry and materials science of cathodic hydrogen absorption and adsorption," Electrochemical Society Proceedings, Vol. 94-21; The Electrochemical Society: Pennington, NJ, 1995.

3. G. Jerkiewicz, 'Electrochemical hydrogen adsorption and absorption. Part 1: Under-potential deposition of hydrogen,' Electrocatal., 1, 179 (2010).

4. R. Gomez, J. M. Orts, A. Bernabe, and J. M. Feliu, 'Effect of temperature on hydrogen adsorption on $\mathrm{Pt}(111), \operatorname{Pt}(110)$, and $\mathrm{Pt}(100)$ Electrodes in $0.1 \mathrm{M}$ $\mathrm{HClO}_{4}$,' J. Phys. Chem. B, 108, 228 (2004).

5. K. Kunimatsu, T. Senzaki, G. Samjesk, M. Tsushima, and M. Osawa, 'Hydrogen adsorption and hydrogen evolution reaction on a polycrystalline Pt electrode studied by surface-enhanced infrared absorption spectroscopy,' Electrochim. Acta, 52, 5715 (2007).

6. E. Gileadi, In "Electrosorption", E. Gileadi, ed. Plenum Press, New York, 1967, pp. 1-18.

7. J. Chun, J. H. Chun, In "Developments in electrochemistry", J. H. Chun, ed. InTech, Rijeka, 2012, Ch. 1, pp. 327 (http://www.intechopen.com/books/developments-inelectrochemistry)

8. J. Chun, N. Y. Kim, J. H. Chun, 'Determination of the adsorption isotherms of hydrogen and deuterium isotopes on a Pt-Ir alloy in LiOH solutions using the phase-shift method and correlation constants', J. Chem. Eng. Data, 55, 5598 (2010). 
9. E. Gileadi, "Electrode kinetics", VCH: New York, 1993, pp. $261-280$.

10. D. W. Oxtoby, H. P. Gillis, N. H. Nachtrieb, "Principles of modern chemistry", 5th ed. Thomson Learning Inc.: New York, 2002, p. 446.

11. J. Y. Chun, J. H. Chun, 'Correction and supplement to the determination of the optimum intermediate frequency for the phase-shift method [Chun et al., Int. J. Hydrogen Energy 30 (2005) 247-259, 1423-1436]', Int. J. Hydrogen Energy, 33, 4962 (2008).
12. J. H. Chun, S. K. Jeon, J. Y. Chun, 'The phase-shift method and correlation constants for determining adsorption isotherms of hydrogen at a palladium electrode interface', Int. J. Hydrogen Energy, 32, 1982 (2007).

13. J. Y. Chun, J. H. Chun, 'A negative value of the interaction parameter for over-potentially deposited hydrogen at Pt, Ir, and Pt-Ir alloy electrode interfaces', Electrochem. Commun., 11, 744 (2009).

14. E. Gileadi, "Electrode kinetics", VCH: New York, 1993, pp. 303-305 